\title{
Lehr- und Lernziele im postgymnasialen Fremdsprachenunterricht in Polen
}

\begin{abstract}
Today's language politics assumes that every citizen should habe a good command of a least two foreign languages. There are three main teaching goals connected with learning foreign languages: pargmatic, cognitive and affective. In the present paper all of three goals haev been in the context of over-secondary school education in Poland.
\end{abstract}

\section{FREMDSPRACHEPOLITIK IN EUROPA UND IHRE BEDEUTUNG FÜR POLEN}

In dem Weißbuch zur allgemeinen und beruflichen Bildung 1995 wird als Ziel festgelegt, dass alle EU-Bürger drei europäische Sprachen, von denen eine ihre Muttersprache ist, beherrschen sollten. Um dieses Ziel zu erreichen muss noch viel in der Fremdsprachenpolitik getan werden, es gibt aber schon heute Länder, in denen die meisten Menschen drei Sprachen beherrschen (laut Eurobarometer-Umfrage im Jahr $2001^{2}$ waren das $26 \%$ der EUBürger).

Auch in Polen hat man die Notwendigkeit einer Reform hinsichtlich der Fremdsprachenpolitik erkannt. Seit der Wende um 1989 wurden in Polen mehrere Schulreformen durchgeführt, darunter eine des polnischen Schulwesens, die am 1. September 2002 in Kraft trat. In Folge der Veränderung der Schuldauer der jeweiligen Schultypen sowie der Einführung des Gymnasiums, entstanden neue Zielgruppen, deren Alter, Interessen und Bedürfnisse neu in Betracht gezogen werden mussten. Es mussten aber nicht nur neue Lehrpläne, sondern auch neue Lehrwerke verfasst werden. Da sich

1 Vgl. http://europa.eu.int/comm/education/policies/lang/languages/lang/reasons_de.html

2 Die Umfrage wurde im Dezember 2000 in allen 15 EU-Mitgliedstaaten durchgeführt, nachzulesen unter: http://europa.eu.int/comm/education/policies/lang/languages/lang/eurobarometer54_en.html 
aber die Veränderungen und der Schulsystemwandel schneller vollzogen, als dass neue Curricula und Lehrbücher verfasst werden konnten, kam es in den neunziger Jahren zu einer solchen Situation, in der die meisten Lehrwerke für Deutsch als Fremdsprache importiert werden mussten, die aber einen geringen Zusammenhang mit den bislang in Polen geltenden Lehrplänen aufwiesen (vgl. Iluk 1998: 8), was wiederum verursachte, dass sich kaum jemand an die Lehrpläne hielt. Somit verloren die Lehrpläne "ihre fundamentale, d.h. steuernde, stabilisierende und innovative Funktion" (Iluk 1998: 8).

Um den Weg aus der Zwickmühle anzubahnen, gab das Bildungsministerium den Lehrern die Möglichkeit, Curricula selbst zu verfassen. Die Curriculumentwicklung und die Lernzielbeschreibung erfolgen heute in Polen auf Grund der vom Bildungsministerium veröffentlichten Programmgrundlage. Die Tätigkeit des Bildungsministeriums bezüglich des Curriculums beschränkt sich auf die Überprüfung und das Zulassungsverfahren.

Der folgende Beitrag stellt einen Umriss der gegenwärtigen Lehr- und Lernziele des Fremdsprachenunterrichts der postgymnasialen Stufe in Polen dar.

\section{LEHR- UND LERNZIELE DES FREMDSPRACHENUNTERRICHTS}

In der didaktischen Diskussion unterscheidet man zwischen den Lehrzielen und den Lernzielen, die selten identisch sind. Die Lehrer bemühen sich um das Erfüllen der curricularen Vorgaben, während die Möglichkeit der Lerner "eigene Zielsetzungen ins Spiel zu bringen" begrenzt bleibt (Krumm 2003b: 116). Auch Doyé unterscheidet zwischen Lernzielen, die sich Menschen für ihr eigenes Lernen setzen und Lehrzielen, die Menschen bei der Steuerung der anderen intendieren (vgl. Doyé 1995: 161).

Da der Fremdsprachenunterricht an den Schulen zu den Pflichtfächern gehört, kann davon ausgegangen werden, dass es sich in der schulischen Realität wesentlich öfter um die Lehr- als die Lernziele handelt (vgl. Krumm 2003b: 116, Doyé 1995: 161).

\subsection{Pragmatische Lehr- und Lernziele}

Die pragmatische Kompetenz gilt heute als primäres Lehrziel und ist von allen drei Lehrzielen am wenigsten umstritten.

Die pragmatischen Lehr- und Lernziele beziehen sich auf die kommunikativen Fertigkeiten, die folgend gegliedert werden (vgl. Storch 1999: 15, Pfeiffer 2001: 152): 
gesprochene Sprache geschriebene Sprache

rezeptiv

produktiv
Hörverstehen

Sprechfertigkeit
Leseverstehen

Schreibfertigkeit

Die Unterscheidung erfolgt nach dem Medium (gesprochene oder geschriebene Sprache) und nach der kommunikativen Grundhaltung, die ein kommunikativ Handelnder einnehmen kann, darunter die Informationsentnahme (rezeptiv) und die Informationsvermittlung (produktiv).

Die vier Kompetenzen, nicht mehr Fertigkeiten „in dem älteren, mehr technischen Sinne" (Doyé 1995: 162), sind eng miteinander verbunden, aber in dem Unterrichtsgeschehen kommen ihnen je nach späterer Relevanz verschiedene Rollen zu.

Doyé (1995: 163f.) und Storch (1999: 25) sprechen von Verwendungsmöglichkeiten der Fremdsprache bei der jeweiligen Gruppe von Lernern, die sich in:

- der Rolle,

- der Situation,

- den Kommunikationsabsichten,

- den Themen,

- der Textsorte,

- dem Medium

äußern. Da die pragmatischen Lehr- und Lernziele des Fremdsprachenunterrichts handlungsorientiert sind, geht die Zielexplizierung von der Situation aus, in der der Lerner vermutlich handeln wird. Dazu gehören der Ort der Sprechhandlung, die Themen, die in dieser Situation angesprochen werden. Es werden auch die Rolle und die sozialen Beziehungen berücksichtigt, darunter Alter, Nationalität (Identitätsmerkmale), ob der Lerner Käufer, Arzt oder Kunden spielt (Funktionsrollen), seine Haltung (affektive Einstellung), Bekanntschaftsgrad und Rangverhältnis (gleichberechtigt, untergeordnet, übergeordnet). Auch die Fertigkeiten, die in dem jeweiligen Unterrichtsabschnitt entwickelt werden sollen, ob der Lerner sich z.B. auf das Sprechen oder Zuhören konzentrieren soll, spielen eine bedeutende Rolle wie die kommunikativen Absichten, ob man einen Wunsch äußert oder eine Bitte ausdrückt.

Aufgrund dieser Kriterien lassen sich "Anhaltspunkte für die Auswahl pragmatisch verwertbarer sprachlicher Mittel gewinnen" (Storch 1999: 26). Die einzelnen Kompetenzen sind komplex und erfordern die Schulung sog. Teilkompetenzen (vgl. Lehrplan u. a. von Boszulak und Ciemnicka 2002), z. B. auf die Sprechfertigkeit setzen sich u.a. die Kenntnis des Wortschatzes, der grammatischen Strukturen und korrekter Aussprache zusammen. Manche Theoretiker stellen die Zerlegung der Kompetenz in untergeordnete Teilkompetenzen in Frage, die meisten jedoch halten eine zeitweilige Be- 
schränkung auf die Teilbereiche, z. B. bei Lernschwierigkeiten, für sinnvoll (vgl. Doyé 1995: 162). Es muss aber im Auge behalten werden, dass nur eine Integration aller vier Kompetenzen kommunikativen Erfolg verspricht.

In Polen hängt das Niveau der sprachlichen Teilkompetenzen, die die Schüler erwerben müssen, von der Schul- und Bildungsstufe ab. Seit der Schulreform 2002 sind auch die zu erreichenden Sprachniveaus mit den im Gemeinsamen Europäischen Referenzrahmen für das Lernen und Lehren von Sprachen festgelegten Sprachniveaus vergleichbar. Die sechs verschiedenen Sprachniveaus führen von geringen Grundkenntnissen (Niveaustufe A1) über die Niveaus A2, B1, B2, C1, bis zum Niveau C2.

In polnischen Lehrplänen, in der Variante $\mathrm{C}^{3}$ soll das Sprachniveau A2 erzielt werden, in der Variante B - B1, in der Variante A - C1. Fast alle Curricula beinhalten eine Liste der „Kann-Beschreibungen" (vgl. Profile deutsch 2002), in der alle sprachlichen Aktivitäten im Einklang mit der Programmgrundlage für jedes Sprachniveau einzeln definiert werden. Und so soll der polnische Schüler z. B. in der mündlichen Produktion in der Variante C imstande sein, kurz und einfach über alltägliche Aktivitäten zu berichten, während er in der Variante B schon die Fähigkeit haben soll, im eigenen Fach- oder Interessenbereich detaillierte Antworten $\mathrm{zu}$ geben, einfache Verhandlungen $\mathrm{zu}$ führen, über andere berichten zu können (vgl. Lehrplan von Boszulak und Ciemnicka 20024, Lewicki 2002, Cwikowska und Wojdat-Niklewska 2002).

Die pragmatischen Lehr- und Lernziele werden von Anfang an im Unterricht vermittelt und im Laufe der Zeit auf allen Lernstufen geschult. Dabei muss unterstrichen werden, dass das kurstragende Element im Schulunterricht die Lehrwerke sind, und diese entscheiden und demnach sich voneinander unterscheiden, wie sie "die curricularen Vorgaben in ein didaktisches Konzept umsetzen" (Storch 1999: 26). Die Lehrer sind diejenigen, die die Wahl eines adäquaten Lehrwerkes für die Einsetzung im Unterricht verantworten.

\subsection{Kognitive Lehr- und Lernziele}

Die kognitiven Lernziele beziehen sich im Allgemeinen auf die Kenntnisse und Einsichten, die im Unterricht gewonnen werden sollen (vgl. Heyd 1991: 37).

${ }^{3}$ In der Programmgrundlage werden drei Varianten für das Lehren einer fremden Sprache in der vierten Bildungsstufe definiert, ausgehend von den Fremdsprachenkenntnissen der Schüler und der Zahl für dieses Fach vorgesehenen Unterrichtsstunden: Variante A (Aufbaukurs, 4-5 Std./Woche, erste Fremdsprache), Variante B (Aufbaukurs, 2-3 Std./Woche, zweite Fremdsprache, oder Grundkurs 4-5 Std./Woche, erste Fremdsprache), Variante C (Grundkurs, 2Std./Woche, zweite Fremdsprache).

${ }^{4}$ Bei den Lehrplänen wird das Jahr angegeben, in dem der Lehrplan von dem Bildungsministerium zugelassen wurde. 
Da der Fremdsprachenunterricht an eine konkrete Sprache gebunden ist, sollen im Unterricht die Kenntnisse über das Zielsprachenland und deren Kultur vermittelt werden. Damit gewinnt die explizite Landeskunde in der kognitiven Dimension an Bedeutung.

Die Erfüllung der Forderung nach Einführung und Realisierung der landeskundlichen Inhalte im Unterricht ist nach Doyé (1995: 163) damit begründet, da "Sprache ein System von Zeichen (ist), und Zeichen haben Form und Inhalt. Niemand kann eine Sprache losgelöst von den Inhalten, die sie bezeichnet, lehren, und jeder sinnvolle Fremdsprachenunterricht gelangt deshalb zwangsläufig dahin, mit der Sprache auch eine andere Kultur zu vermitteln". Doyé (1995: 163) bezeichnet das als semiotische Begründung, neben der pädagogischen Begründung, wo die Erweiterung des geistigen Horizonts der Schüler über nationale Grenzen hinaus angestrebt werden, die Beschäftigung mit anderen Kulturen ihm den Blick für anderes, Fremdes öffnen und ihn vor ethnozentrischem Denken bewahren solle.

Um einen Überblick über die kognitiven Lernziele zu verschaffen, bedienen wir uns der Einteilung von Storch (1999: 28), der das kognitive Wissen folgenden Bereichen zuordnet:

- das landeskundliche Wissen (über "Land und Leute"),

- das soziokulturelle Wissen (befähigt in einem fremden Land situations- und partnergerecht adäquat zu handeln),

- das pragmatische Wissen (über die Form bestimmter Textsorten, den Einsatz bestimmter Redemittel),

- das Wissen über das Lernen von Fremdsprachen (Lernstrategien).

Das implizite Ziel der Landeskunde im Fremdsprachenunterricht ist das $\mathrm{zu}$ verstehen, was uns fremd und oft dadurch feindlich scheint. Wenn der Schüler die Möglichkeit bekommt in den fremdkulturellen Alltag Einsicht zu gewinnen, die Denk- und Lebensweise, Werte, Überzeugungen, Einstellungen der anderen sprachkulturellen Vertreter kennen zu lernen und mit eigenen zu vergleichen, kann das zur Revidierung seiner Ansichten führen und damit direkt zur Realisierung des Lernziels der interkulturellen Kompetenz beitragen.

Die landeskundlichen Inhalte, die in die Lehrpläne für Deutsch als Fremdsprache (vgl. Machowiak 2002, Brewinska und Obidniak 2002, Lewicki 2002, Boszulak und Ciemnicka 2002) explizit aufgenommen werden betreffen:

- Informationen zu den deutschsprachigen Ländern (im Bereich der Geschichte, Geographie, des politischen Systems, der Wirtschaft und Kultur),

- Informationen zu Sitten und Bräuchen, Lebensweisen, Sprachen, Dialekten,

- Realienkunde (z. B. Sehenswürdigkeiten, Währung),

- Wissen über Kultur (Literatur, Musik, Kunst). 
Leider werden Themenvorschläge zur Erschließung der soziokulturellen Wirklichkeit der deutschsprachigen Länder nur Schülern jener Klassen gemacht, die in Klassen mit erweiterten Fremdsprachenstundenzahl lernen. Anstatt also die Schüler von Anfang an um die interkulturelle Komponente $\mathrm{zu}$ bereichern, wird ihnen zu Verstehen gegeben, dass sie sprachlich noch zu schwach sind um über meistens für sie interessante Themen zu sprechen, was leider oft, wenn auch indirekt, ihre Motivation beeinflusst.

Die kognitive Kompetenz umfasst aber nicht nur Vermittlung von neuen Kenntnissen.

$\mathrm{Zu}$ Beginn jedes Lernens verfügen die Lerner über ein bestimmtes Vorwissen über das Zielsprachenland und deren Bewohner, zu denen die Lerner "von vornherein nicht immer die pädagogisch erwünschten Einstellungen und Werthaltungen haben" (Iluk 2000: 41). Die nicht selten schwierige Aufgabe des Lehrers besteht darin, die Lerner auf diese aufmerksam zu machen. Die Schüler sollen mit ihren Einstellungen auseinander setzen lernen.

Jedoch wie Neuner (2004: 85) bemerkt, "jede Gesellschaft entwickelt übergreifende Leitvorstellungen zu der Frage, wie die nachkommende Generation durch Erziehung in der Zeit der allgemeinen Schulpflicht 'auf das Leben' vorbereitet werden soll". Es geht dabei nicht nur um kognitive und pragmatische Ziele, sondern auch um affektive Ziele, darunter "welche ,Werte und Normen' einer Gemeinschaft an die kommende Generation weiter gegeben und welche Haltungen und Einstellungen ausgeprägt werden sollen" (Neuner 2004: 86).

\subsection{Affektive Lehr- und Lernziele}

Affektive Lehr- und Lernziele haben lange Zeit in der fachdidaktischen Diskussion keine Berücksichtigung gefunden. Erst das oberste Ziel des Fremdsprachenunterrichts, die Befähigung zur interkulturellen Kommunikation, machte auf die emotionale Dimension der Lehrziele aufmerksam. Die drei Haltungen und Einstellungen (Offenheit, Toleranz und Kommunikationsbereitschaft) „in Verbindung mit der Vermittlung von gründlichen Kenntnissen über die anderen Kulturen, macht den Fremdsprachenunterricht zu einem bedeutenden Bestandteil der politischen Erziehung in der Schule" (Doyé 1995: 165).

Offenheit bedeutet weitgehende Freiheit von Vorurteilen gegenüber Menschen und Sachverhalten der anderen Kultur. Nach Doyé (1995: 164) wäre viel gewonnen, wenn es gelingen würde, Menschen durch ihren Fremdsprachenunterricht dahin zu führen, dass sie Angehörigen anderer Kulturen mit der gleichen Offenheit begegnen wie ihrer eigenen Kultur. 
Toleranz bedeutet „das andere gelten zu lassen neben der eigenen Art zu leben und die Welt zu sehen" (Doyé 1995: 164). Iluk (2000: 42) unterscheidet hier u. a. Empathie für das fremdkulturelle Individuum, das Verständnis für soziale, wirtschaftliche und politische Ursachen von Ethnozentrismus und kulturelle Konflikte, die Fähigkeit Widersprüchlichkeit ertragen, sowie kulturadäquat mit den Widersprüchen und Konflikten umgehen zu können.

Der letzte Schritt ist nach Doyé die Kommunikationsbereitschaft als Haltung desjenigen, der „angesichts der erlebten Andersartigkeit von Phänomenen der fremden Kulturen und aufgrund seiner Neigung, sie als gleichwertig zu akzeptieren, bereit ist, (...) in eine Kommunikation (...) einzutreten" (Doyé 1995: 164).

Die Lerner sollen nicht mehr im Laufe ihrer Ausbildung bloß die sprachlichen Fertigkeiten beherrschen oder explizites Wissen über die fremde Sprache aneignen. Das neue interkulturelle Konzept zielt auf cultural awareness (vgl. Krumm 2003a: 142, Neuner 2004: 93, Quetz 2003: 124), das die oben genannten Einstellungen und Haltungen voraussetzt.

Cultural awareness bedeutet nicht nur die Auseinandersetzung mit der fremden Welt. Neuner betont, dass die „Beschäftigung mit der fremden Welt, die mit der jeweiligen Fremdsprache verbunden ist, auch Rückwirkung auf die Wahrnehmung der eigenen Welt [hat], sie erweitert und verändert das Bewusstsein von der eigenen Welt, umfasst also auch einen Prozess der Bedeutungsaushandlung von eigener Identität" (Neuner 2004: 93).

\subsection{Allgemeine Bildungsziele}

In den Schulen gilt es jedoch noch außer den pragmatischen, kognitiven und affektiven Zielen allgemeine Bildungsziele zu verfolgen, um in den Schülern entsprechende ethische Haltung zu entwickeln. In der Programmgrundlage des neusprachlichen Unterrichts für die dreijährigen reformierten Lyzeen werden u.a. zu Aufgaben der Schule die fachübergreifenden Erziehungsziele der Selbständigkeit und Verantwortungsbewusstsein für sich und andere, des Selbstbewusstseins und des Glaubens an eigene Möglichkeiten, Wertschätzung der eigenen wie der anderen Kulturen angerechnet (vgl. Doyé 1995: 165, Hoffmann 2000: 92f., Pfeiffer 2001a: 153, Lehrplan von Boszulak und Ciemnicka 2002, Lehrplan von Lewicki 2002 ).

Aus den Lernzielen resultieren konkrete Forderungen nach Inhalten, die während des Unterrichts vermittelt werden sollten.

Hoffmann (2000: 93) listet folgende Lehrinhalte auf:

a) Integration von Kenntnissen und praktischen Fertigkeiten, die der Schüler im Rahmen des Unterrichts in verschiedenen Fächern erworben hat, 
b) Erweiterung der sprachlichen Kompetenz durch den Kontakt mit authentischen schriftlichen und mündlichen Äußerungen, mit gleichzeitiger Berücksichtigung verschiedener sprachlicher Register, des formellen und nichtformellen Stils, sowie literarischer Texte des Landes (bzw. des Raumes) der unterrichteten Sprache,

c) Einbeziehung der kulturellen Thematik des Landes (bzw. des Raumes) der unterrichteten Sprache und des Vaterlandes (kontrastive Kulturkunde).

\section{ABSCHLIESSENDE BEMERKUNGEN}

In der Diskussion um die Lehr- und Lernziele des Fremdsprachenunterrichts darf nicht vergessen werden, dass die genaue Festlegung der Ziele zwar eine gute Grundlage für unterschiedlichste Prüfungen bietet und somit die Transparenz der Fertigkeiten und Inhalte sichert. Andererseits wäre vielleicht in Zukunft zu überlegen, ob solch eine genaue Festlegung der Lehrund Lernziele an den Lehrern die bloße Erfüllung der Lehrpläne nicht erzwingt und das auf Kosten der immer wieder neu diskutierten Forderung nach mehr Autonomie im Unterricht.

Bevor die neu geplante Reform ${ }^{5}$ eingeführt wird, sollte vielleicht in Betracht gezogen werden, dass kaum ein Lehrer an Autonomie im Unterricht denken wird, wenn ihm selbst die Hände gebunden sind.

\section{BIBLIOGRAPHIE}

Christ, I., 2003. Staatliche Regelungen für den Fremdsprachenunterricht: Curricula, Richtlinien, Lehrpläne, in: Bausch, K.-R. / Christ, H. / Krumm, H.-J. (Hg.): Handbuch Fremdsprachenunterricht, Tübingen: Francke: 71-76.

Doyé, P., 1995. Lehr- und Lernziele In: Bausch, K.-R. / Christ, H. / Krumm, H.-J. (Hg.): Handbuch Fremdsprachenunterricht, Tübingen: Francke: 161-166.

Hoffmann, M., 2000. Vielleicht ein Projekt? In: Glottodidactica XXVIII: 91-101.

Heyd, G., 1991. Deutsch lehren. Grundwissen für den Unterricht in Deutsch als Fremdsprache. Diesterweg: Frankfurt a. M.

Iluk, J., 1998. Entwicklung der Sprachfertigkeiten aus der Sicht der neuesten Fremdsprachencurricula. Katowice: Wydawnictwo Uniwersytetu Sląskiego.

Iluk, J., 2000. Erzieherische Ziele aus der Sicht neuerer internationaler Fremdsprachencurricula, In: DaF, Heft 1: 41-47.

Komorowska, H., 1993. Podstawy Metodyki Nauczania Języków Obcych, Warszawa: EDE-Poland.

Krumm, H.-J., 2003a. Curriculare Aspekte des interkulturellen Lernens und der interkulturellen Kommunikation In: Bausch, K.-R. / Christ, H. / Krumm, H.-J. (Hg.): Handbuch Fremdsprachenunterricht, Tübingen: Francke: 138-144.

Krumm, H.-J., 2003b. Lehr- und Lernziele, in: Bausch, K.-R. / Christ, H. / Krumm, H.-J. (Hg.): Handbuch Fremdsprachenunterricht, Tübingen: Francke: 116-120.

${ }^{5}$ Siehe mehr dazu unter: http://www.isp.org.pl/podstawa/ 
Neuner, G., 2004. Schulischer Fremdsprachenunterricht und Fremdsprachenpädagogik. In: Badstübner-Kizik, C. / Rozalowska-Źądlo, R. / Uniszewska, A. (Hg.): Sprachen lehren. Sprachen lernen. Nauczanie i uczenie się języków obcych. Festschrift für Professor Halina Stasiak zum 70. Geburtstag. Gdansk: Wydawnictwo Uniwersytetu Gdanskiego: 85-96.

Pfeiffer, W., 2001. Nauka jzzyków obcych. Od praktyki do praktyki. Poznań: Wagros.

Quetz, J., 2003. Fremdsprachliches Curriculum. In: Bausch, K.-R. / Christ, H. / Krumm, H.-J. (Hg.): Handbuch Fremdsprachenunterricht, Tübingen: Francke: 121-126.

Storch, G., 1999. Deutsch als Fremdsprache - eine Didaktik, München: Wilhelm Fink Verlag.

Zimmermann, G., 1995. Das sprachliche Curriculum. In: Bausch, K.-R. / Christ, H. / Krumm, H.-J. (Hg): Handbuch Fremdsprachenunterricht, Tübingen: Francke: 135-141.

\section{LEHRPLÄNE}

Boszulak, U. / Ciemnicka, E: Uniwersalny program nauczania języka niemieckiego dla liceum ogólnoksztalcacego, liceum profilowanego $i$ technikum. Poziom podstawowy $i$ rozszerzony. Hueber Polska. DKOS-4015-186/02.

Cwikowska, B. / Wojdat-Niklewska, A.: Program nauczania języka niemieckiego dla klas I-III liceum ogólnokształcq̨cego, liceum profilowanego oraz 4-letniego technikum (wariant B lub C). Wydawnictw.

LektorKlett. DKOS-4015-127/02.

Lewicki, R.: Poznaj jezyk sasiada. Program nauczania języka niemieckiego dla liceum ogólnoksztatcqcego, liceum profilowanego i technikum. Kurs podstawowy. Wydawnictwo Szkolne PWN. DKOS4015-56/02.

Machowiak, E. D.: Program nauczania języka niemieckiego dla liceum ogólnokształcącego, liceum profilowanego i technikum. Kurs kontynuacyjny. Wydawnictwo Szkolne PWN. DKOS-4015$71 / 02$. 